\title{
China's community-based strategy of universal preconception care in rural areas at a population level using a novel risk classification system for stratifying couples' preconception health status
}

Qiongjie Zhou ${ }^{1,2,3}$, Shikun Zhang ${ }^{4 *}$, Qiaomei Wang ${ }^{4}$, Haiping Shen ${ }^{4}$, Weidong Tian ${ }^{5}$, Jingqi Chen ${ }^{5}$, Ganesh Acharya ${ }^{3,6}$ and Xiaotian Li $\mathrm{Li}^{1,2,7^{*}}$

\begin{abstract}
Background: Preconception care (PCC) is recommended for optimizing a woman's health prior to pregnancy to minimize the risk of adverse pregnancy and birth outcomes. We aimed to evaluate the impact of strategy and a novel risk classification model of China's "National Preconception Health Care Project" (NPHCP) in identifying risk factors and stratifying couples' preconception health status.

Methods: We performed a secondary analysis of data collected by NPHCP during April 2010 to December 2012 in 220 selected counties in China. All couples enrolled in the project accepted free preconception health examination, risk evaluation, health education and medical advice. Risk factors were categorized into five preconception risk classes based on their amenability to prevention and treatment: A-avoidable risk factors, B- benefiting from targeted medical intervention, $\mathrm{C}$-controllable but requiring close monitoring and treatment during pregnancy, D-diagnosable prenatally but not modifiable preconceptionally, X-pregnancy not advisable. Information on each couple's socio-demographic and health status was recorded and further analyzed.
\end{abstract}

Results: Among the 2,142,849 couples who were enrolled to this study, the majority (92.36\%) were from rural areas with low education levels (89.2\% women and $88.3 \%$ men had education below university level). A total of 1463266 (68.29\%) couples had one or more preconception risk factors mainly of category A, B and C, among which $46.25 \%$ were women and $51.92 \%$ were men. Category A risk factors were more common among men compared with women $(38.13 \%$ versus $11.24 \% ; P=0.000)$.

Conclusions: This project provided new insights into preconception health of Chinese couples of reproductive age. More than half of the male partners planning to father a child, were exposed to risk factors during the preconception period, suggesting that an integrated approach to PCC including both women and men is justified. Stratification based on the new risk classification model demonstrated that a majority of the risk factors are avoidable, or preventable by medical intervention. Therefore, universal free PCC can be expected to improve pregnancy outcomes in rural China.

Keywords: Preconception care, Preconception health, Risk stratification, Reproductive health, Population-based study, Rural China, Universal preconception care, Community-based care

\footnotetext{
*Correspondence: yiping791129@163.com; xiaotianli555@163.com

${ }^{4}$ The National Health and Family Planning Commission, Beijing, China

'Obstetrics and Gynecology Hospital of Fudan University, 419 Fangxie Road,

Shanghai 200011, China

Full list of author information is available at the end of the article
} 


\section{Background}

Preconception care (PCC) is defined as interventions that aim to identify and, when possible, modify the biomedical, behavioral, and social risks to optimize woman's health before pregnancy with the aim of improving pregnancy outcomes [1]; In 2014, Centers for Disease Control and Prevention (CDC) and the Office of Population Affairs published clinical recommendations, "Providing Quality Family Planning Services" (QFP), and recognized PCC as a critical component of health care for women of reproductive age [2].

The purpose of PCC is to optimize a woman's health prior to pregnancy and promote healthy behavior during pregnancy to reduce the incidence of adverse birth outcomes [3]. It is reported that an estimated 300,000 women die globally as a result of pregnancy-related conditions [4]. The prevalence of birth defects in China is around 5.6\%, and there are nearly 900000 new cases annually according to the official Report on Prevention of Birth Defects in China published in 2012 [5]. Health services provided to the couples of reproductive age, such as family planning, folic acid supplementation [6], genetic counseling, chronic disease management, immunizations, treatment of sexually transmitted infections, and interventions promoting healthier lifestyle, including those directed against alcohol, tobacco, and substance abuse [7] seem to have a positive effect. There is growing evidence that effective treatment of maternal diabetes and hypertension during the preconception period reduces adverse maternal and neonatal outcomes [8-10]. Avoiding unintended pregnancy through PCC could avert $44 \%$ maternal mortality [11]. Moreover, the effect of PCC on women with a history of previous adverse infant outcome, such as preterm birth, low birth weight, stillbirth or major birth defect, appears to be meaningful [12].

Even though the benefits of PCC have been well established [13, 14], integrating PCC into regular family planning services still remains a challenge for some providers [15]. Poor organization of health services' delivery systems, lack of comprehensive PCC programs, limited awareness among future parents about the availability and benefits of PCC and that of physicians about the necessity and effectiveness of PCC are apparent barriers affecting delivery and uptake of PCC $[16,17]$.

PCC in China has been insufficient and inadequate, especially in rural areas, despite the fact that facility-based strategy on reducing neonatal mortality had a significant impact on the Millennium Development Goal 4, and with a rapid economic development there have been improvements in population health in recent decades [18]. Therefore, the National Health and Family Planning Commission of the People's Republic of China(NHFPC)launched the "National Preconception Health Care
Project" (NPHCP) in 2010, focusing on rural areas and providing free PCC for the couples of reproductive age [19]. In this project, relevant preconception risk factors were classified according to their amenability to prevention and treatment. The objective of our study was to evaluate the impact of strategy and risk classification model of China's NPHCP in identifying risk factors and stratifying the preconception health status of men and women of reproductive age.

\section{Methods}

\section{Data source and study design}

We conducted a secondary analysis of data collected within the framework of NPHCP during April 2010 to December 2012 to investigate the characteristics of preconception risk factors among married Chinese women and men of reproductive age. Methodological details of the project have been described previously [20-22]. Briefly, the study covered 220 counties in China. Selected rural counties in all provinces and the urban counties that wanted to participate in this project were included in this population-based prospective cohort study.

NHFPC established the implementation and quality control standards for this program $[20,21]$. Local community staff investigated the conception plans of the couples, and those planning to conceive within the next six months were enrolled and invited to attend a free health examination. Professional doctors specially trained in obstetrics, genetic and other related specialties provided necessary medical advice to the couples. NHFPC has drafted and published the consultation guide for common preconception health problems. All couples enrolled accepted a free preconception health examination, risk evaluation, health education and medical advice based on the risk factors. A written informed consent was obtained from each participant, and this study was approved by the Institutional Review Board of the Chinese Association of Maternal and Child Health Studies [20, 21].

Preconception examination included (1) a medical history: current medical illness and use of any medication, family history of hypertension, diabetes, congenital or genetic diseases in the first-degree relatives, life style, dietary habits and exposure to environmental and occupational hazards; (2) physical examination: height, weight, blood pressure, heart rate, palpation of thyroid gland, auscultation of the heart and lungs, abdominal palpation, examination of the limbs and the spine; (3) clinical laboratory tests: genital swabs for microbiological culture and sensitivity, gonococcus and chlamydia test, hemoglobin and full blood count, urine for bacteriology and culture, blood type, serum glucose, liver, renal function and thyroid function tests, hepatitis B serology, syphilis test, TORCH (toxopasma, 
rubella virus, cytomegalovirus, and herpes simplex virus) screen, and gynecological ultrasound; (4) past medical history: hypertension, diabetes, cardiac diseases, immune system diseases, renal diseases and other chronic diseases; (5) past obstetric history including history of induced abortion, spontaneous abortion, live birth, stillbirth, neonatal death, fetal abnormality, preterm birth and multiple pregnancy. Trained staff regularly recorded and entered the information into the NHFPC database.

\section{Preconception risk evaluation and classification model}

The aim of the preconception health examination was to identify all the risk factors as far as possible, and treat accordingly. Therefore, instead of assessing the degree of exposure, we developed a preconception risk classification system based on their amenability to prevention and treatment according to Preconception Health Examination and Risk Evaluation Guides (Science and Technology Division of NHFPC) (Table 1). Risk factors were categorized into five preconception risk classes: A-avoidable risk factors, B-benefiting from targeted medical intervention before conception, $\mathbf{C}$-controllable but requiring close monitoring and treatment during pregnancy, $\mathbf{D}$-diagnosable prenatally but the risk factor not modifiable preconceptionally, X-pregnancy not advisable. The couples with category $\mathrm{X}$ risk factor were advised to use appropriate contraception and were considered in further analysis. Participants with missing or incomplete records were excluded from analysis.

\section{Statistical analysis}

Statistical analysis was performed using SPSS statistical software version 15.0 (SPSS, System for Windows, Chicago, USA). Data are presented as number (\%) and mean \pm standard deviation (SD). For comparing groups, we used independent samples $t$-test for continuous variable and $\chi^{2}$ test for categorical variables. All $P$-values were two-tailed, and a $P<0.05$ was considered to be statistically significant.

Table 1 Definition of "ABCDX" category of preconception risk factors

\begin{tabular}{lll}
\hline Category & Definition & Risk factors \\
\hline A & $\begin{array}{l}\text { Avoidable risks, i.e. they could be avoided though health } \\
\text { education and eliminating work place hazards etc. }\end{array}$ & $\begin{array}{l}\text { Maternal: smoking, alcohol consumption, exposure to toxins, } \\
\text { radiation, noise, pesticide, organic solvent, heavy metal, inadequate } \\
\end{array}$ \\
& $\begin{array}{l}\text { nutrition (no intake of meat and egg, no intake of fresh vegetables, } \\
\text { raw meat eating habit) }\end{array}$ \\
& $\begin{array}{l}\text { Paternal: smoking, alcohol, consumption, exposure to toxins, } \\
\text { radiation exposure, noise, pesticide, organic solvent, high temperature, } \\
\end{array}$ & preputial ring, inadequate nutrition (no intake of meat and egg, \\
& no intake of fresh vegetables, raw meat eating habit)
\end{tabular}

B Benefiting from targeted medical intervention,

\begin{abstract}
Maternal: anemia ${ }^{a}$, bacterial vaginitis, candida infection, gonorrhoea, trichomoniasis, Toxoplasma gondii infection (lgM positive), gingival hemorrhage, history of psychological disorder;

Paternal: abnormal liver function, abnormal renal function, spermatic cord varicocele, hypertension, congenital heart disease, history of chronic renal disease history, cancer, epilepsy, or psychological disorder

Maternal: Thrombocytopeniab, abnormal liver function, abnormal renal function, abnormal TSH, HBs-Ag positive, HBe-Ag positive, cytomegalovirus IgM positive, chlamydia positive, syphilis screening positive, Rh negative, history of gynecological diseases, preterm birth, diabetes, congenital heart disease, hypertension, malignancy, chronic renal disease, reported epilepsy, tuberculosis, use of narcotics; Paternal: HBs antigen positive, HBe antigen positive, syphilis screening positive, use of narcotics, thyroid disease
\end{abstract}

Maternal: Maternal birth defect, history of previous child with birth defects, mental retardation, history of recurrent abortion, stillbirth, or neonatal death, family history of Mediterranean anemia, G6PD deficiency, Albinism, Down's syndrome, visual impairment; hearing impairment;

Paternal: Paternal birth defect, mental retardation, family history of neonatal death, Mediterranean anemia, G6PD deficiency, Albinism, Down's syndrome, hemophilia, family history of visual impairment or hearing impairment 
Table 2 Socio-demographic characteristics of women in different preconception risk factor classification categories

\begin{tabular}{llllllll}
\hline & & No risk factors & A & B & C & D \\
\hline Age & $\leq 25$ & $24.7 \%$ & $21.6 \%$ & $26.3 \%$ & $22.4 \%$ & $14.7 \%$ & $21.3 \%$ \\
& $25-30$ & $46.3 \%$ & $47.4 \%$ & $46.7 \%$ & $44.1 \%$ & $38.5 \%$ & $46.0 \%$ \\
& $30-35$ & $19.5 \%$ & $21.2 \%$ & $18.2 \%$ & $20.9 \%$ & $27.1 \%^{*}$ & $20.1 \%$ \\
& $\geq 35$ & $9.6 \%$ & $9.8 \%$ & $8.7 \%$ & $12.6 \%$ & $19.8 \%^{*}$ & $12.6 \%^{*}$ \\
\multirow{2}{*}{ Area } & Rural area & $93.9 \%$ & $89.6 \%$ & $94.0 \%$ & $94.0 \%$ & $94.3 \%$ & $92.2 \%$ \\
Race & Han & $92.7 \%$ & $91.6 \%$ & $92.5 \%$ & $88.7 \%$ & $84.0 \%^{*}$ & $84.2 \%^{*}$ \\
Education & Secondary school or lower & $71.5 \%$ & $64.8 \%^{*}$ & $69.4 \%$ & $71.8 \%$ & $77.4 \%$ & $75.5 \%$ \\
& High school & $18.7 \%$ & $19.7 \%$ & $18.7 \%$ & $17.1 \%$ & $13.8 \%^{*}$ & $16.6 \%$ \\
& College or higher & $9.8 \%$ & $15.5 \%^{*}$ & $11.8 \%$ & $11.1 \%$ & $8.8 \%$ & $8.0 \%$ \\
\hline
\end{tabular}

${ }^{*} P$ value $<0.05$ compared with those women having no risk factors

\section{Results}

General characteristics of the study population

During April 2010 to December 2012, a total of 22.42 million married Chinese couples planning to conceive within the six months were recruited to the study from 220 different counties. After excluding those with incomplete medical records and lost to follow-up, data from 2,142,849 couples were available for analysis. NPHCP targeted couples of reproductive age mainly from rural areas, and covered most areas, regions, and ethnicities from all provinces of mainland China. 92.36\% couples were from rural areas and $89.2 \%$ women and $88.3 \%$ men had education below university level. Other socio-demographic details of the participants are presented in Tables 2 and 3.

\section{Preconception risk factor classification}

As demonstrated in Tables 2 and 3, category D risk was more common among couples in the age group 30-35 years and $>35$ years $(P<0.05)$. There were no significant differences between rural areas and cities in both couples in terms of risk factor categories. Proportionally, more women of non-Han ethnicity were classified in category $\mathrm{D}$ and $\mathrm{X}$ compared to those with no risk factors, while there was no difference in that ratio among men. Women with category A, and men with category B and $\mathrm{D}$ risk factors had higher education levels $(P<0.05)$.

\section{Distribution preconception risk factors}

Distribution of the participants in different preconception risk categories is presented in Table 4. Among 2,142,849 couples, $46.25 \%$ women had preconception risks, mainly of category A, B and C. 9.80\% women had category A risks including alcohol consumption (3.4\%), inadequate protein intake (1.36\%) and exposure to noise (1.18\%). 14.83\% women were had category B risks, such as anemia $(8.40 \%)$, gingival hemorrhage $(3.57 \%)$ and vaginitis $(2.29 \%)$. Moreover, $23.5 \%$ of women had category C risks, such as thyroid dysfunction (6.34\%), HBV infection (4.76\%), history of gynecological diseases (3.41\%) and/or category D risks, such as history of spontaneous abortion $(2.66 \%)$ and adverse pregnancy history (1.12\%). On the other hand, $51.92 \%$ of couples had paternal risks, and $38.13 \%$ of them had category A risk factors including alcohol (29.61\%) and smoking (29.07\%) (Table 4).

Table 3 Socio-demographic characteristics of men in different preconception risk categories

\begin{tabular}{|c|c|c|c|c|c|c|c|}
\hline & & No risk factors & A & $B$ & C & D & $\bar{x}$ \\
\hline \multirow[t]{4}{*}{ Age } & $\leq 25$ & $9.2 \%$ & $10.2 \%$ & $10.3 \%$ & $9.1 \%$ & $4.5 \%{ }^{*}$ & - \\
\hline & $25-30$ & $43.1 \%$ & $44.5 \%$ & $44.9 \%$ & $45.5 \%$ & $38.6 \%{ }^{*}$ & - \\
\hline & $30-35$ & $28.8 \%$ & $27.6 \%$ & $28.0 \%$ & $28.1 \%$ & $33.0 \%{ }^{*}$ & - \\
\hline & $\geq 35$ & $18.9 \%$ & $17.6 \%$ & $16.8 \%$ & $17.3 \%$ & $23.9 \%^{*}$ & - \\
\hline Area & Rural area & $92.6 \%$ & $92.1 \%$ & $90.1 \%$ & $92.2 \%$ & $85.7 \%$ & - \\
\hline Race & Han & $92.7 \%$ & $91.3 \%$ & $88.4 \%$ & $92.3 \%$ & $91.1 \%$ & - \\
\hline \multirow[t]{3}{*}{ Education } & Secondary school or lower & $69.3 \%$ & $69.0 \%$ & $63.1 \%$ & $66.4 \%$ & $61.9 \%$ & - \\
\hline & High school & $20.0 \%$ & $19.2 \%$ & $20.1 \%$ & $20.2 \%$ & $16.9 \%$ & - \\
\hline & College or higher & $10.6 \%$ & $11.8 \%$ & $16.8 \%^{*}$ & $13.4 \%$ & $21.3 \%^{*}$ & - \\
\hline
\end{tabular}

${ }^{*}: P$ value $<0.05$ compared with those men having no risk factors 
Table 4 Distribution of preconception risk factors among women and men in different preconception risk categories

\begin{tabular}{|c|c|c|c|}
\hline \multirow{2}{*}{$\begin{array}{l}\text { Risk factors } \\
\text { Maternal }\end{array}$} & \multirow[t]{2}{*}{ Total number (\%) } & Risk factors & \multirow[t]{2}{*}{ Total number $(\%$} \\
\hline & & Paternal & \\
\hline \multicolumn{4}{|l|}{ A } \\
\hline Alcohol consumption & $72,808(3.40 \%)$ & Alcohol consumption & $634,547(29.61 \%)$ \\
\hline No intake meat and egg & $29,126(1.36 \%)$ & Smoking & $622,834(29.07 \%)$ \\
\hline Noise exposure & $25,267(1.18 \%)$ & Preputial ring & $84,659(3.95 \%)$ \\
\hline \multirow[t]{6}{*}{ Others $^{a}$} & \multirow[t]{6}{*}{$82,739(3.86 \%)$} & Noise exposure & $48,399(2.26 \%)$ \\
\hline & & Pesticide exposure & $26,753(1.25 \%)$ \\
\hline & & No intake meat and egg & $25,857(1.21 \%)$ \\
\hline & & Organic solvent exposure & $24,211(1.13 \%)$ \\
\hline & & High temperature exposure & $23,388(1.09 \%)$ \\
\hline & & Others $^{i}$ & $41,492(1.94 \%)$ \\
\hline \multicolumn{4}{|l|}{ B } \\
\hline Anemia $^{\mathrm{b}}$ & $179,941(8.40 \%)$ & Abnormal liver function & $152,862(7.13 \%)$ \\
\hline Gingival hemorrhage & $76,595(3.57 \%)$ & Abnormal renal function & $26,107(1.22 \%)$ \\
\hline Vaginitis $^{c}$ & $49,162(2.29 \%)$ & Others $^{j}$ & $9,973(0.47 \%)$ \\
\hline Others $^{d}$ & $12,257(0.57 \%)$ & & \\
\hline \multicolumn{4}{|l|}{ c } \\
\hline Abnormal TSH & $135,958(6.34 \%)$ & HBs antigen positive & $135,958(5.87 \%)$ \\
\hline HBs-Ag positive & $101,970(4.76 \%)$ & HBe antigen positive & $101,970(1.68 \%)$ \\
\hline Gynecological diseases history & $73,107(3.41 \%)$ & Others ${ }^{k}$ & $10,111(0.47 \%)$ \\
\hline Abnormal renal function & $57,680(2.69 \%)$ & & \\
\hline Abnormal liver function & $54,192(2.53 \%)$ & & \\
\hline Thrombocytopenia $^{\mathrm{b}}$ & $31,522(1.47 \%)$ & & \\
\hline HBe-Ag positive & $31,459(1.47 \%)$ & & \\
\hline Others ${ }^{\mathrm{e}}$ & $132,582(6.19 \%)$ & & \\
\hline \multicolumn{4}{|l|}{ D } \\
\hline Spontaneous abortion history & $57,060(2.66 \%)$ & Others' & $4,305(0.20 \%)$ \\
\hline Adverse pregnancy history ${ }^{f}$ & $23,878(1.12 \%)$ & & \\
\hline Others $^{9}$ & $8,873(0.41 \%)$ & & \\
\hline \multicolumn{4}{|l|}{$x$} \\
\hline Others $^{h}$ & $5,259(0.25 \%)$ & & \\
\hline
\end{tabular}

${ }^{a}$ including 20,647 not eating fresh vegetables $(0.96 \%), 16,435$ pesticide exposure $(0.77 \%), 16,049$ organic solvent exposure $(0.75 \%), 12,540$ radiation exposure $(0.59 \%), 9,717$ smoking $(0.45 \%), 5,582$ raw meat eating habit $(0.26 \%)$ and 1,765 heavy metal exposure $(0.08 \%)$

${ }^{\mathrm{b}}$ Anemia referred to hemoglobin ranging from $60-109 \mathrm{~g} / \mathrm{L}$

'Vaginitis included 27,657 Candida infection, 11,398 Bacterial vaginitis and 10,107 Trichomonasis

${ }^{d}$ including 10,107 Trichomoniasis $(0.47 \%)$, 7,672 Toxoplasma gondiilgM positive $(0.36 \%), 4,545$ Gonococcal infection $(0.21 \%)$ and 40 history of psychological disease $(0.00 \%)$ eincluding 20,705 Rh negative (0.97\%), 9,290 Cytomegalovirus IgM positive (0.43\%), 9,266 Chlamydia positive (0.43\%), 8,482 Syphilis screening positive (0.40\%), 4,395 history of preterm birth $(0.21 \%), 14,383$ diabetes $(0.67 \%), 1,830$ reported hypertension $(0.09 \%), 1,655$ reported history of tuberculosis $(0.08 \%), 1,392$ anesthetic drug use $(0.07 \%), 1,327$ congenital heart disease $(0.06 \%), 1,018$ reported tumor history $(0.05 \%), 897$ reported chronic renal disease history $(0.04 \%)$ and 882 reported epilepsy history $(0.04 \%)$

${ }^{\mathrm{f}}$ Adverse pregnancy history included 16,824 with history of stillbirth and 7,054 with history of birth defects

gincluding 4,515 with birth defects $(0.21 \%), 1,527$ family history of neonatal death $(0.07 \%), 1,416$ mental retardation $(0.07 \%)$, 923 family history of Mediterranean anemia $(0.04 \%), 254$ family history of G6PD deficiency $(0.01 \%), 138$ family history of Albinism $(0.01 \%), 92$ family history of Down's syndrome $(0.00 \%), 5$ family history of hearing impairment $(0.00 \%), 2$ family history of mental retardation $(0.00 \%)$ and 1 family history of visual impairment $(0.00 \%)$

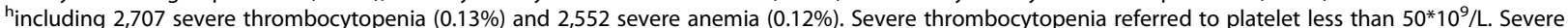
anemia referred to hemoglobin less than $60 \mathrm{~g} / \mathrm{L}$

'including 18,726 not eating fresh vegetables $(0.87 \%), 9,734$ radiation exposure $(0.45 \%), 9,454$ raw meat eating habit $(0.44 \%)$ and 3,578 exposure to heavy metals $(0.17 \%)$ jincluding 5,325 spermatic cord varicocele $(0.25 \%), 2,432$ hypertension $(0.11 \%), 1,052$ congenital heart disease $(0.05 \%), 598$ chronic renal disease history $(0.03 \%)$, 404 epilepsy $(0.02 \%), 159$ history of cancer $(0.01 \%)$ and 3 history of psychological disease $(0.00 \%)$



'including 2,344 with birth defects $(0.11 \%), 658$ family history of neonatal death $(0.03 \%), 603$ family history of Mediterranean anemia $(0.03 \%), 274$ family history of G6PD deficiency $(0.01 \%), 248$ mental retardation $(0.01 \%), 138$ family history of Albinism $(0.01 \%), 78$ family history of Down's syndrome $(0.00 \%), 4$ family history of hearing impairment $(0.00 \%)$, 1 family history of hemophilia $(0.00 \%)$ and 1 family history of visual impairment $(0.00 \%)$ 


\section{Discussion}

This nation-wide free preconception care project targeting rural areas in China used an integrated model of PCC including both women and men. A novel classification system was used to classify risk factors based on their amenability to prevention and treatment, which stratified couples in five different risk categories. More than $68 \%$ of couples with conception plans within the next six months had one or more risk factors, and nearly $40 \%$ of these risk factors could be potentially modified by intervention before or during pregnancy. Approximately $23 \%$ of risk factors among women were in category A and B, whereas among men the figure was $45 \%$. Avoidable risk factors were more common among men compared with women suggesting that men may have riskier behavior than women, with almost $30 \%$ of men reporting consumption of alcohol and smoking.

Our study revealed that preconception risk evaluation in couples with plans to conceive within six months could be meaningful as nearly two-thirds of the recruited couples had preconception risk factors, and 23\% maternal risk factors were in category $\mathrm{A}$ and $\mathrm{B}$, and thereby potentially avoidable or modifiable preconceptionally by health education, medical intervention and life style changes. More importantly, a similar situation was observed regarding paternal risk factors. Almost $45 \%$ of the male partners consumed alcohol or smoked, which may lead to passive smoking by women, a fact often ignored in preconception care. Some European countries have preconception care recommendations for women with chronic diseases, such as diabetes and epilepsy, but guidelines are heterogeneous and recommendations for healthy women and men are fragmented and inconsistent [22]. Our results further enforce the need for an integrated approach to PCC that includes both women and men.

A more innovative and integrated approach to PCC for both women and men is needed for achieving optimal reproductive health status before pregnancy and better pregnancy outcomes [23, 24]. Preconception health promotion may be useful in eliminating some of the Category A and B risk factors before pregnancy. However, some risk factors, such as smoking, alcohol and substance abuse, would require longer term strategies to achieve sustained amelioration. A more comprehensive health promotion strategy during pregnancy would be required for managing other risk categories to achieve better pregnancy outcomes.

The preconception risk classification system used in this big population-based study was practical for stratifying preconception health status of the couples, and helpful in organizing targeted educational and health care interventions, and identifying need for referral. The risk classification was based on existing risk factors during the preconception period and categorized by whether it could be prenatally avoided or modified during the preconception period or prenatally. As preconception risks may vary from prenatal risks, Considering different methods and timing of intervention is important. Nearly half of the risk factors identified were avoidable or preventable by medical intervention during the preconception period in this study, allowing for a window of opportunity for personalized lifestyle modification and health care to achieve better pregnancy outcome. Despite the evidence supporting the value and importance of PCC [25], it is reported that there is lack of sufficient research attention to clinical PCC service delivery, and a more detailed consideration of the practicalities of implementing PCC within contemporary women's health care is required [26]. This integrated universal free PCC service provided in rural China could be a promising model if its positive impact on pregnancy outcomes could be demonstrated in future.

Our study does have some limitations. Follow-up of risk modifications was not included in this study, so the impact of preconception risk classification on the health status of the couple could not be assessed. Prevalence of adverse pregnancy history and chronic disease history in couples planning pregnancy might have been underestimated as this was based on self-reporting and recall bias cannot be excluded.

\section{Conclusions}

This project provided new insights into preconception health of Chinese couples of reproductive age. More than half of the male partners planning to father a child were exposed to risk factors during the preconception period, suggesting that an integrated approach to PCC including both women and men is justified. Stratification based on the new risk classification model demonstrated that a majority of the risk factors are avoidable or preventable by medical intervention. Therefore, universal free PCC can be expected to improve pregnancy outcomes in rural China.

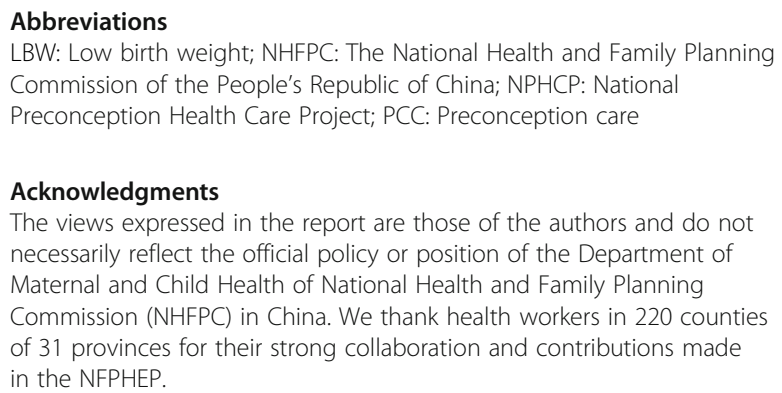

\section{Acknowledgments}

The views expressed in the report are those of the authors and do not necessarily reflect the official policy or position of the Department of Maternal and Child Health of National Health and Family Planning Commission (NHFPC) in China. We thank health workers in 220 counties of 31 provinces for their strong collaboration and contributions made in the NFPHEP.

\section{Funding}

This study was funded by the Chinese Association of Maternal and Child Health Studies (AMCHS-2014-4). 


\section{Availability of data and materials}

Dataset analyzed in this study was based on the national database and public access to the database is closed. Zhang Shikun gave the administrative permission to access the database on behalf of National Health and Family Planning Commission of the People's Republic of China (NHFPC).

\section{Authors' contributions}

ZQ and LX carried out the statistical analysis and drafted the manuscript. GA interpreted data and drafted the manuscript. ZS, WQ, SH and LX participated in the design of the study and coordination. TW and CJ performed the statistical analysis. All authors read and approved the final manuscript.

\section{Competing interests}

The authors declare that they have no competing interests.

\section{Consent for publication}

Not applicable

\section{Ethics approval and consent to participate}

This study was approved by the Institutional Review Board of Chinese Association of Maternal and Child Health Studies. A written informed consent was obtained from each participant, as consent to participate.

\section{Author details}

'Obstetrics and Gynecology Hospital of Fudan University, 419 Fangxie Road, Shanghai 200011, China. ${ }^{2}$ The Shanghai Key Laboratory of Female Reproductive Endocrine-Related Diseases, Shanghai, China. ${ }^{3}$ Women's Health and Perinatology Research Group, Department of Clinical Medicine, UiT - The Arctic University of Norway, Tromsø, Norway. ${ }^{4}$ The National Health and Family Planning Commission, Beijing, China. ${ }^{5}$ School of Life Sciences, Institute of Biostatistics, Fudan University, Shanghai, China. 'Department of Clinical Science, Intervention and Technology (CLINTEC), Karolinska Institute, Stockholm, Sweden. ${ }^{7}$ Institute of Biomedical Sciences, Fudan University, Shanghai, China

Received: 19 February 2016 Accepted: 2 December 2016

Published online: 28 December 2016

\section{References}

1. Johnson K, Posner SF, Biermann J, Cordero JF, Atrash HK, Parker CS, et al. Recommendations to improve preconception health and health care- United States. A report of the CDC/ATSDR Preconception Care Work Group and the Select Panel on Preconception Care. MMWR Recomm Rep. 2006;55(6):1-23.

2. Robbins CL, Loretta G, Zapata LB, Carter MW, Christina L, Nancy M-S, et al. Preconception Care in Publicly Funded U.S. Clinics That Provide Family Planning Services. Am J Prev Med. 2016;51(3):336-43.

3. Inskip HM, Crozier SR, Godfrey KM, Borland SE, Cooper C, Robinson SM, et al. Women's compliance with nutrition and lifestyle recommendations before pregnancy: general population cohort study. BMJ. 2009:338:b481.

4. Alkema L, Chou D, Hogan D, Zhang S, Moller AB, Gemmill A, et al. Global, regional, and national levels and trends in maternal mortality between 1990 and 2015, with scenario-based projections to 2030: a systematic analysis by the UN Maternal Mortality Estimation Inter-Agency Group. Lancet. 2016:387:462-74.

5. Ministry of Health of People's Republic of China. The report on the prevention of birth defects in China. 2012. Available online at: www.gov.cn/ gzdt/2012-09/12/content_2223371.htm. Accessed 17 Jan 2016.

6. De-Regil LM, Fernández-Gaxiola AC, Dowswell T, Peña-Rosas JP. Effects and safety of periconceptional folate supplementation for preventing birth defects. Cochrane Database Syst Rev. 2010;6(10):CD007950.

7. Crawford C. AAFP urges family physicians to integrate preconception care into patient visits. Ann Fam Med. 2016;14:180-1.

8. Seely EW, Ecker J. Clinical practice. Chronic hypertension in pregnancy N Engl J Med. 2011;365(5):439-46.

9. Bentley-Lewis R. Gestational diabetes mellitus: an opportunity of a lifetime. Lancet. 2009;373(9677):1738-40.

10. Black RE, Victora CG, Walker SP, Bhutta ZA, Christian P, de Onis M, et al. Maternal and Child Nutrition Study Group. Maternal and child undernutrition and overweight in low-income and middle-income countries. Lancet. 2013;382(9890):427-51.
11. Saifuddin A, Qingfeng L, Li L, Tsui AO. Maternal deaths averted by contraceptive use: an analysis of 172 countries. Lancet. 2012;380:111-25.

12. Wahabi HA, Alzeidan RA, Bawazeer GA, Alansari LA, Esmaeil SA. Preconception care for diabetic women for improving maternal and fetal outcomes: a systematic review and meta-analysis. BMC Pregnancy Childbirth. 2010;10:63

13. Beckmann MM, Widmer T, Bolton E. Does preconception care work? Aust N Z J Obstet Gynaecol. 2014;54(6):510-4.

14. Luton D, Forestier A, Courau S, Ceccaldi PF. Preconception care in France. Int J Gynaecol Obstet. 2014;125(2):144-5.

15. M'hamdi HI, Voorst SF, Pinxten W, Hilhorst MT, Steegars EA. Barriers in the uptake and delivery of preconception care exploring the views of care providers. Matern Child Health J. 2016. Epub ahead of print.

16. M'hamdi HI, van Voorst SF, Wim P, Hilhorst MT, Eric AP. Steegers. Barriers in the uptake and delivery of preconception care: exploring the views of care providers. Matern Child Health J. 2016. Epub ahead of print.

17. Poels M, Koster MP, Boeije HR, Franx A, van Stel HF. Why do women not use preconception care? A systematic review on barriers and Facilitators. Obstet Gynecol Surv. 2016;71(10):603-12.

18. Feng XL, Guo GF, Hipgrave D, Zhu J, Zhang L, Song L, et al. China's facilitybased birth strategy and neonatal mortality: a population-based epidemiological study. Lancet. 2011;378:1493-500.

19. Zhou Q, Acharya G, Zhang S, Wang Q, Shen H, Li X. New perspective on universal preconception care in China. Acta Obstet Gynecol Scand. 2016; 95(4):377-81.

20. Zhang SK, Wang QM, Shen HP. Design, implementation and objective of free "National Preconception Health Care Project in China. Natl Med J China. 2015;95(3):162-5.

21. Wang QM, Zhang M, Zhang SK, Hu M, Liu N, Kang XX, et al. Establishment of quality control system of free "National Preconception Health Care Project in China. Natl Med J China. 2015;95(3):166-8.

22. Jill Shawe ID, Maria E, Hanne Kristine H, Margareta L, Pierpaolo M, et al. Preconception care policy, guidelines, recommendations and services across six European countries: Belgium (Flanders), Denmark, Italy, the Netherlands, Sweden and the United Kingdom. Eur J Contracept Reprod Health Care. 2015;20:77-87.

23. Michelle SF, Karla D, Brian J. The future of preconception care in the United States: multigenerational impact on reproductive outcomes. Ups J Med Sci. 2016;19:1-5.

24. Anna B, Gunilla L. Preconception health and care (PHC) — a strategy for improved maternal and child health. Ups J Med Sci. 2016;20:1-6.

25. Braspenningx S, Haagdorens M, Blaumeiser B, Jacquemyn Y, Mortier G. Preconceptional care: a systematic review of the current situation and recommendations for the future. Facts Views Vis Obgyn. 2013;5(1):13-25.

26. Steel A, Luckec J, Reida R, Adams J. A systematic review of women's and health professional's attitudes and experience of preconception care service delivery. Fam Pract. 2016; Epub ahead of print.

\section{Submit your next manuscript to BioMed Central and we will help you at every step:}

- We accept pre-submission inquiries

- Our selector tool helps you to find the most relevant journal

- We provide round the clock customer support

- Convenient online submission

- Thorough peer review

- Inclusion in PubMed and all major indexing services

- Maximum visibility for your research

Submit your manuscript at www.biomedcentral.com/submit
) Biomed Central 